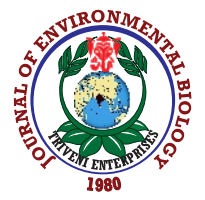

\title{
In silico binding analysis of human CD40 ligand mimetic molecule, 3-(dimethylamino)-1-phenyl-1- propanone hydrochloride (3-DPH), with CD40 receptor molecules of various mammalian species
}

\author{
S. Sivagami', R. Rathna', S. Nagavignesh', N.V. Ghone ${ }^{2}$ and M. Sivanandham ${ }^{1 *}$ \\ ${ }^{1}$ Department of Biotechnology, Sri Venkateswara College of Engineering (Autonomous), Sriperumbudur-602 117, India \\ 'Department of Chemical Engineering \& Biotechnology, Sri Venkateswara College of Engineering (Autonomous), Sriperumbudur-602 117, India \\ *Corresponding Author Email : msiva@svce.ac.in
}

\section{Abstract}

Aim: To investigate the binding of human CD40 ligand (CD40L) mimetic molecule, 3-(dimethylamino)-1-phenyl-1-propanone hydrochloride (3-DPH), with CD40 receptor (CD40R) molecules of Homo sapiens, Cavia porcellus, Cricetulus griseus, Macaca mulatta, Mus musculus, Oryctolagus cuniculus, Papio anubis and Rattus norvegicus species using bioinformatics tool.

Methodology: Three-dimensional structures of CD40Rs and CD40Ls for various mammalian species were generated using the published crystal structure of human CD40 receptor-ligand complex by homology modelling using SWISS-MODEL tool. Furthermore, human CD40L mimetic molecule, 3$\mathrm{DPH}$ was docked against the generated CD40R of various mammalian species using AUTODOCK 4.2 .

Results: Docking studies revealed that documented HIS78 and GLN79 residues of human CD40R were the key interaction residues, which interacted with human CD40L and 3-DPH. The CD40Rs of $H$. sapiens, C. porcellus, C. griseus, M. mulatta, $M$. musculus, $O$. cuniculus, $P$. anubis, and $R$. norvegicus bind with 3-DPH with a binding energy $-4.67,-5.22,-5.19,-4.62,-4.85$, $4.63,-4.51$, and $-4.86 \mathrm{kcal} / \mathrm{mol}$, respectively.

Interpretation: Molecular docking studies provide crucial insight into the binding affinity and interaction of 3-DPH at the active site of CD40R of the respective mammalian species. 0 . cuniculus and $M$. musculus species were found to be appropriate animal models for further evaluation of the therapeutic effect of human CD40L mimetic molecule.

Key words: 3-DPH, Animal model, CD40R, CD40L, Homo sapeins, Molecular docking

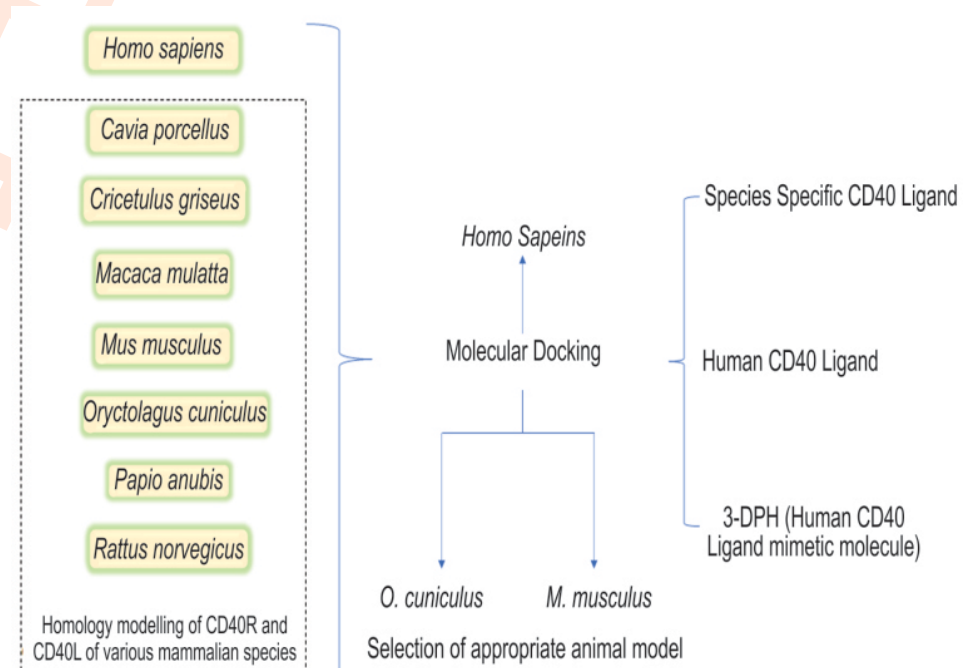

How to cite : Sivagami, S., R. Rathna, S. Nagavignesh, N.V. Ghone and M. Sivanandham: In silico binding analysis of human CD40 ligand mimetic molecule, 3(dimethylamino)-1-phenyl-1-propanone hydrochloride (3-DPH), with CD40 receptor molecules of various mammalian species. J. Environ. Biol., 42, 186-191 (2021). 


\section{Introduction}

Interaction of CD40 ligand (CD40L) with CD40 receptor (CD40R) plays a significant role in humoral and cellular immune responses (Kawabe et al., 2011; Kooten and Banchereau, 1996). Selective stimulation or inhibition of immune response by this molecule provides an opportunity for the development of immunotherapeutic agents to treat pathogenic disease and immunological disorders (Durie et al., 1994; Yi and Bishop, 2015). Studies demonstrated that engagement of CD40R with CD40L induces activation and proliferation of B cells (Brenner et al., 1998), which augments the immune response. Croft et al. (2013) reported that blocking CD40R-CD40L interaction showed high efficacy in experimental models and clinical trials of immunological disorder.

Also, clinical studies on the inhibition of CD40R-CD40L interactions using monoclonal antibodies (Imai et al., 2007) and ligands (Silvian et al., 2011) exhibited a promising outcome for immunological diseases (karpusas et al., 1995). However, prolonged inhibition by these monoclonal antibodies and ligands leads to thrombolytic complications (Koyama et al., 2004; Kawai et al., 2000). Therefore, further studies of these therapeutic compounds are underway to refine these molecules (Couzin, 2005). Peptidomimetics is another custom-designed antigenic peptide mimetic molecule which aid in blocking the costimulatory pathway. Fournel et al. (2005) developed a small C3-symmetric CD40L mimetic molecule. However, CD40L peptidomimetics suffer from low binding (Allen et al., 2005; Kitagawa et al., 2005).

Therefore, studies on organic non-peptide mimetic molecules show biological activities similar to that of the original compound is an active area of research for finding potent drugs (Guarnieri, 2015; Franz, 2007; Galloway et al., 2010). With this purpose, three organic human CD40L mimetic molecules, namely NSC25: Benzoyl(trimethyl)-5-azane (ABTC), NSC76: NBenzhydryl benzamide (NBB) and NSC89: 3-(Dimethylamino)-1phenyl-1-propanone hydrochloride (3-DPH) (Vani and Sivanandham, 2008) were computationally designed. These compounds have reported augmenting the immune response by switching immunoglobin type $\lg \mathrm{M}$ to $\lg \mathrm{G}$ and to induce in vitro proliferation of human B cells (Vani et al., 2014). Of these compounds, 3-DPH is known as mono mannich bases reported with anti-microbial activity, anti-cancer activity, and other medical applications (Roman, 2015).

Further, in vitro and in-vivo studies are needed to evaluate the therapeutic potential of this human CD40L mimetic molecule. Prior to in-vitro and in-vivo studies, it is prudent to perform molecular docking analysis to identify the active site residues and their key interactions in determining appropriate species for further investigation of human CD40L mimetic molecule. In view of the above, this study aimed to understand the interaction of human CD40L mimetic molecule, 3-DPH with CD40R molecules of Homo sapiens, Cavia porcellus, Cricetulus griseus, Macaca mulatta, Mus musculus, Oryctolagus cuniculus, Papio anubis and Rattus norvegicus species using molecular docking approach.

\section{Materials and Methods}

CD40R and CD40L sequence analysis: The FASTA sequence of CD40L and CD40R proteins of $H$. sapiens (Human; Acc. No.: Ligand: NP_000065.1, Receptor: EAW75762.1), C. porcellus (Guinea pig; Acc. No.: Ligand: XP_003464759.1, Receptor: XP 013005826.1), C. griseus (Hamster; Acc. No.: Ligand: XP_027289243.1, Receptor: XP_003500573.1), M. mulatta (Rhesus monkey; Acc. No.: Ligand: NP 001028011.1, Receptor: EHH19629.1), M. musculus (Mouse; Acc. No.: Ligand: NP_035746.2, Receptor: AAB08705.1), O. cuniculus (Rabbit; Acc. No.: Ligand: NP_001243710.1, Receptor: XP_002721245.1), P. anubis (Baboon; Acc. No.: Ligand: XP_003918394.1, Receptor: NP_001306202.1), and $R$. norvegicus (Rat; Acc. No.: Ligand: NP_445805.1, Receptor: NP 599187.1) were obtained from the National Centre for Biotechnology Information (NCBI, USA). Multiple sequence alignment and phylogenetic tree construction were carried out for CD40R and CD40L sequences using Clustal Omega webform: https://www.ebi.ac.uk/Tools/msa/clustalo/ (Sievers et al., 2011; Patnaik et al., 2019) to find the sequence and active site residue similarity across $H$. sapiens, C. porcellus, C. griseus, M. mulatta, M. musculus, $O$. cuniculus, $P$. anubis and $R$. norvegicus.

Various mammalian species receptor and ligand modelling: CD40R and CD40L structures for the selected mammalian species were not available at RCSB Protein Data Bank (http://www.rcsb.org/). Hence, the human CD40 receptor-ligand (CD40-CD154) complex (PDB ID:3QD6), which is identical to $\mathrm{CD} 40 \mathrm{R}$ of various mammalian species, were selected for further studies. In 3QD6, residues 126-131 and 146-190 of CD40R were not included due to poor visibility in the electron density map. However, conserved active site residues were not part of the missing region. The $3 \mathrm{D}$ structure of $\mathrm{CD} 40 \mathrm{R}$ and $\mathrm{CD} 40 \mathrm{~L}$ of various mammalian species were modelled using the SWISS-MODEL server in SIB Bioinformatics Resource Portal. The complete CD40 receptor and ligand amino acid sequence of various mammalian species were retrieved from NCBI (https://www.ncbi.nlm.nih.gov/). The sequence similarities were performed to identify homologs using Clustal Omega (https://www.ebi.ac.uk/Tools/msa/clustalo/). For homology modelling, CD40-CD154 co-crystal structure (3QD6) chain R and chain A were selected as a template.

Molecular docking: Molecular docking was carried out to study the binding mode of ligands at the active site of CD40R. AUTODOCK 4.2 (The Scripps Research Institute, USA) was used to calculate the free energy of atoms binding between the CD40R of various mammalian species and CD40L mimetic molecules (Goodsell and Olson, 2000; Morris et al., 1996; Morris et al., 1998). A $5 \AA$ grid was built surrounding the binding pockets formed between the various mammalian species CD40R and their ligand, human CD40L and 3-DPH mimetic molecule. Three-dimensional grids of interaction energy for all possible atom types that were already present in the AUTODOCK default parameter set was calculated. These grid maps were of dimension $60 \times 60 \times 60$ points, with the spacing of $0.375 \AA$ yielding a receptor model that included atoms within $0.5 \AA$ of grid centre. The Lamarckian Genetic Algorithm was opted to search for the best conformers. During docking, an empirical AUTODOCK scoring function was determined as described by Park et al. (2014). At the end of docking, molecular interactions between the ligand and receptor 
were analysed, and their docked conformations were studied using PyMol (https://pymol.org/2/). The interactions of CD40RCD40L conformations, including hydrogen bonds and bond lengths, were analysed using PyMol software. Furthermore, the best conformation was determined using the lowest docked energy during docking search.

\section{Results and Discussion}

A multiple sequence alignment of amino acid sequence of the CD40 receptor and ligand across various mammalian species was analysed using Clustal Omega. The CD40R sequence of $H$. sapiens, $C$. porcellus, C. griseus, M. mulatta, $M$. musculus, $O$. cuniculus, $P$. anubis, and $R$. norvegicus showed a sequence similarity of $100 \%, 67 \%, 60 \%, 92 \%, 61 \%, 69 \%, 92 \%$ and $59 \%$ with $3 \mathrm{QD} 6 \mathrm{R}$ chain, respectively. The evolutionary relationship of CD40R and $\mathrm{CD} 40 \mathrm{~L}$ sequences across the mammalian species were analysed using a phylogenetic tree. Fig. $1 \mathrm{~A}$ depicts the evolutionary relationship of CD40R amino acid sequence across the mammalian species.

Fig. 1B depicts the phylogenetic tree illustrating the evolutionary relationship of CD40L amino acid sequence of various mammalian species with CD40L of $H$. sapiens. The CD40L sequence of $H$. sapiens, $C$. porcellus, C. griseus, $M$. mulatta, M. musculus, $O$. cuniculus, $P$. anubis, and $R$. norvegicus showed a sequence similarity of $100 \%, 84 \%, 74 \%$, $98 \%, 77 \%, 66 \%, 98 \%$ and $76 \%$ with 3QD6 A chain, respectively. The CD40 receptor and ligand phylogenetic tree revealed a clear demarcation into two clades from the respective eight input sequences. The results showed that all the CD40R and CD40L sequences across various mammalian species are evolutionarily related to each other. CD40R amino acid sequence of the mammalian species $H$. sapiens, C. porcellus,
C. griseus, M. mulatta, M. musculus, $O$. cuniculus, $P$. anubis, and $R$. norvegicus were retrieved from NCBI.

Human CD40R has three domains, namely extracellular, cytoplasmic, and transmembrane domains containing 173, 62, and 42 amino acids. Among them, the extracellular domain of CD4OR is mainly involved in the interaction with CD40L. Therefore, the residues involved in CD40R-CD40L interaction (higher than $5 \AA$ and less than $7 \AA$ ) were THR70, TRP71, ASN72, ARG73, GLU74, HIS76, CYS77, HIS78, GLN79, TYR82, ASP84, ASN86, THR112, GLU114, ALA 115, GLU117, SER118 of CD40R interacts with ARG203 and ARG207 of CD40L (van Kooten and Banchereau, 2000). Analysing CD40R amino acid sequence across these mammalian species leads to the identification of common conserved site residues and conserved active site residues.

Homology modelling predicts three-dimensional protein structures by using sequence similarity with its homologous template protein sequence. The safe zone for homology modelling is a prediction of the protein structure with less than 150 residues and sequence identity higher than 50\% (Krieger et al., 2003). For homology models, where sequence identity was between $50 \%$ and $90 \%$, the root-mean-square deviation of the atomic coordinates (RMSD) can be around $1.5 \AA$ (Venselaar et al., 2009). Therefore, the choice of mammalian species having the best sequence and active site similarity were identified and selected for homology modelling. From the alignment, the corresponding CD40R active site residues similarity percentage were $78 \%, 61 \%, 89 \%, 78 \%, 94 \%, 89 \%$ and $72 \%$ for $C$. porcellus, C. griseus, M. mulatta, M. musculus, $O$. cuniculus, $P$. anubis, and $R$. norvegicus, respectively with human CD40R. Active site residues of human CD40L were similar across the various mammalian species.

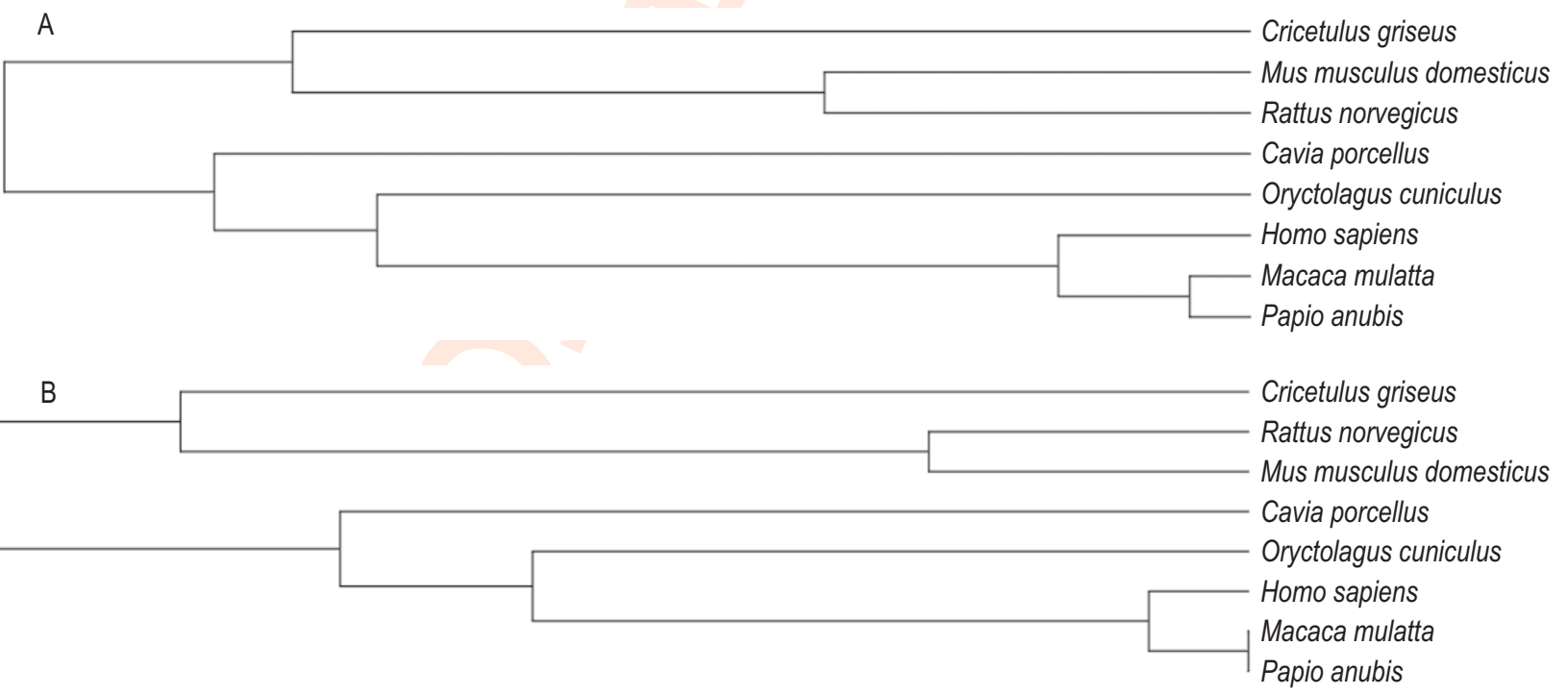

Fig. 1: Phylogenetic analysis across mammalian species such as H. sapien, C. porcellus, C. griseus, M. mulatta, M. musculus, O. cuniculus, P. anubis and $R$. norvegicus (A) CD40 receptor (B) CD40 ligand (Tree scale: 0.01 ). The 0.01 reference scale marks $1 \%$ estimated sequence variance. 

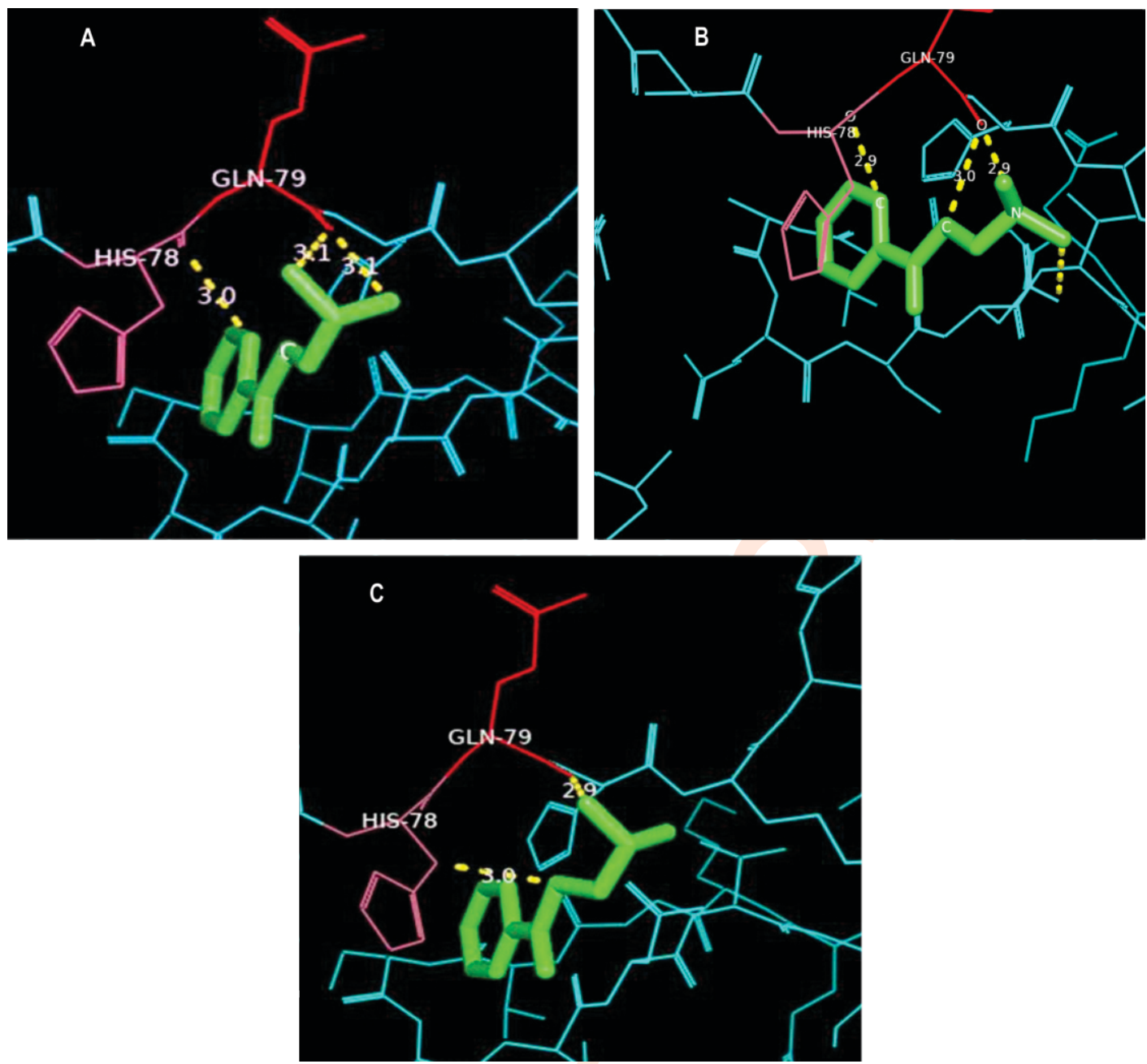

Fig. 2: Interaction of human CD40L mimetic molecule, 3-(dimethylamino)-1-phenyl-1-propanone hydrochloride (3-DPH), with CD40R (A) Residues involved in the interaction of 3-DPH with $H$. sapiens CD40R (B) Residues involved in the interaction of 3-DPH with M. musculus CD40R (C) Residues involved in the interaction of 3-DPH with O. cuniculus CD40R.

In the present study, 3-DPH, a human CD40L mimetic molecule, was subjected to docking with CD40R of $H$. sapiens, $C$. porcellus, C. griseus, M. mulatta, M. musculus, O. cuniculus, $P$. anubis, and $R$. norvegicus using AUTODOCK. Among the previously reported active site residues, histidine and glutamic acids made significant bonds with key residues in the ligand molecules to which the importance is very much explored (Vani and Sivanandham, 2008; van Kooten and Banchereau, 2000; Singh et al., 1998). The major interacting sites and binding energy of CD40R of the selected mammalian species, when interacting with its respective CD40L and human CD40L, are listed in Table 1. Docking results showed that CD40R-CD40L interaction of $H$. sapiens occurred through HIS78 of CD40R and ARG203 of CD40L with a binding energy of $-3.81 \mathrm{kcal} \mathrm{mol}^{-1}$. Similar amino acid-mediated interactions were observed in CD40R of M. mulatta and 0 . cuniculus with their respective ligands. Likewise, the CD40R of M. musculus and P. anubis interacts with the same residue against human CD40L. Thus, this data suggests that the CD40R-CD40L interaction of $M$. mulatta, $M$. musculus, $O$. cuniculus and $P$. anubis showed the highest similarity with $H$. sapiens.

Interacting sites and binding energy for 3-DPH with CD40 receptors of $H$. sapiens and other mammalian species are listed in Table 2. Among the interactions, an aromatic motif of histidine in the receptor is known for its peculiar interactions in proteins (Liao et al., 2013). The low binding energy indicates stable nature and effective binding interactions of the receptor with the ligand molecule. Similarly, a negative docking score is associated with minimum energy that predicts an effective docking of the receptor and ligand. 3-DPH interacts with $H$. sapiens CD40R at HIS78 and GLN79 (Fig. 2A) using oxygen atom of carbonyl group with binding energy $-4.67 \mathrm{kcal} \mathrm{mol}^{-1}$. Similar binding interactions were 
Table 1: Interacting sites and binding energy for CD40 receptor and CD40 ligand interactions

\begin{tabular}{|c|c|c|c|c|c|}
\hline \multicolumn{2}{|c|}{ Interacting molecules } & \multirow{2}{*}{$\begin{array}{l}\text { Interacting } \\
\text { residues of } \\
\text { CD40R }\end{array}$} & \multirow{2}{*}{$\begin{array}{l}\text { Interacting } \\
\text { residues of } \\
\text { CD40L }\end{array}$} & \multirow{2}{*}{$\begin{array}{l}\text { Bond } \\
\text { length } \\
(\AA)\end{array}$} & \multirow{2}{*}{$\begin{array}{c}\text { Binding } \\
\text { energy } \\
\left(\mathrm{kcal} \mathrm{mol}^{-1}\right)\end{array}$} \\
\hline CD40R & CD40L & & & & \\
\hline \multirow[t]{3}{*}{ Human } & Human & HIS78 & ARG203 & 2.9 & -3.81 \\
\hline & & GLN79 & ARG207 & 2.5 & \\
\hline & C. porcellus & ASN72 & ARG206 & 2.7 & -4.20 \\
\hline \multirow{4}{*}{ C. porcellus } & & THR70 & ARG206 & 3.2 & \\
\hline & & TYR78 & ARG203 & 3.0 & \\
\hline & Human & HIS82 & ARG207 & 3.0 & -4.11 \\
\hline & & GLU117 & ARG207 & 2.7 & \\
\hline \multirow[t]{3}{*}{ M. musculus } & M. musculus & GLU74 & ARG202 & 2.6 & -2.58 \\
\hline & Human & GLU74 & ARG203 & 2.7 & -4.30 \\
\hline & & HIS78 & ARG203 & 2.7 & \\
\hline \multirow[t]{3}{*}{ O. cuniculus } & O. cuniculus & HIS78 & ARG203 & 2.7 & -4.01 \\
\hline & Human & HIS76 & ARG207 & 3.3 & -3.98 \\
\hline & & HIS78 & ARG207 & 2.8 & \\
\hline
\end{tabular}

Table 2: Interacting site and binding energy for 3-DPH organic CD40L mimetic molecules with various mammalian CD40R

\begin{tabular}{|c|c|c|c|c|}
\hline CD40R & $\begin{array}{l}\text { Interacting residues } \\
\text { of CD40R }\end{array}$ & Interacting groups & $\begin{array}{l}\text { Bond } \\
\text { length }(\AA ̊)\end{array}$ & $\begin{array}{l}\text { Binding energy } \\
\left(\mathrm{kcal} \mathrm{mol}^{-1}\right)\end{array}$ \\
\hline Human & $\begin{array}{l}\text { HIS78 } \\
\text { GLN79 }\end{array}$ & O-atom with carbonyl group & $\begin{array}{l}3.0 \\
3.1\end{array}$ & -4.67 \\
\hline C. porcellus & $\begin{array}{l}\text { TYR78 } \\
\text { VAL79 }\end{array}$ & O-atom with carbonyl group & $\begin{array}{l}2.9 \\
2.7\end{array}$ & -5.22 \\
\hline M. musculus & $\begin{array}{l}\text { HIS78 } \\
\text { GLN79 }\end{array}$ & $\begin{array}{l}\text { O-atom with carbonyl group } \\
\mathrm{N} \text {-atom with carbonyl group }\end{array}$ & $\begin{array}{l}2.9 \\
2.9\end{array}$ & -4.85 \\
\hline O. cuniculus & $\begin{array}{l}\text { HIS78 } \\
\text { GLN79 }\end{array}$ & $\begin{array}{l}\text { C-atom with carbonyl group } \\
\text { O-atom with carbonyl group }\end{array}$ & $\begin{array}{l}3.0 \\
2.9\end{array}$ & -4.63 \\
\hline
\end{tabular}

found in M. mulatta, M. musculus (Fig. 2B), O. cuniculus (Fig. 2C), $P$. anubis and $R$. norvegicus with a binding energy of $-4.62,-4.85$, $4.63,-4.51$ and $-4.86 \mathrm{kcal} \mathrm{mol}^{-1}$, respectively.

The CD40R of C. griseus (TYR78 and GLN79) and C. porcellus (TYR78 and VAL79) interacted with 3-DPH at different sites with a binding energy of -5.19 and $-5.22 \mathrm{kcal} \mathrm{mol}^{-1}$, respectively. Since dendritic cells induce cytokine production for augmenting immune response through CD40R-CD40L interactions (Cella et al., 1996) and unavailability of dendritic cell-stimulating cytokines such as GMCSF and TNF makes $M$. mulatta and $P$. anubis (Herodin et al., 2005) unsuitable animal models. Based on the interactions of CD40R of selected mammalian species with their respective ligand, human CD40L and 3-DPH, O. cuniculus and M. musculus showed better interactions similar to that of humans.

CD40R-CD40L interaction is one of the most critical immunomodulatory mechanisms in the development of host immunity. 3-DPH, a CD40 ligand mimetic molecule, can play an important therapeutic role in several immunological disorders related to CD40R-CD40L interaction. The current molecular docking studies of 3-DPH with CD40R of 0 . cuniculus and M. musculus species revealed that HIS78 and GLN79 are the key interacting residues, likewise in the $3 \mathrm{DPH}$ interaction with human CD40R. Owing to this similarity and low binding energy, $O$. cuniculus and M. musculus were found to be appropriate animal models for further evaluation of therapeutic effect of human CD40L mimetic molecule.

\section{Acknowledgment}

The authors duly acknowledge the Department of Biotechnology, Sri Venkateswara College of Engineering for their support throughout the research.

\section{Add-on Information}

Authors' contribution: S. Sivagami, R. Rathna: Experimentation and Manuscript preparation; S. Nagavignesh: Result analysis; V.N. Ghone: Result analysis and manuscriprt preparation; M. Sivanandham: conception, design of experiment, result analysis and manuscript preparation.

Research content: The research content is original and has not been published elsewhere 
Ethical approval: NotApplicable

Conflict of interest: The authors declare that there is no conflict of interest.

\section{Data from other sources: NotApplicable}

Consent to publish: All authors agree to publish the paper in Journal of Environmental Biology.

\section{References}

Allen, S.D., S.V. Rawale, C.C. Whitacre and P.T. Kaumaya: Therapeutic peptidomimetic strategies for autoimmune diseases: Costimulation blockade. J. Pept. Res., 65, 591-604 (2005).

Brenner, B., E. Gulbins, U. Koppenhoefer, F. Lang and O. Linderkamp: Dual function of the CD40 ligand (gp39) for T-and B-lymphocytes. Pediatr. Res., 44, 428-428 (1998).

Cella, M., D. Scheidegger, K. Palmer-Lehmann, P. Lane, A. Lanzavecchia and G. Alber: Ligation of CD40 on dendritic cells triggers production of high levels of interleukin- 12 and enhances $T$ cell stimulatory capacity: TT help via APC activation. J. Exp. Med., 184, 747-752 (1996).

Couzin, J.: Drug discovery. Magnificent obsession. Science, 307, 1712-1715(2005)

Croft, M., C.A. Benedict and C.F. Ware: Clinical targeting of the TNF and TNFR superfamilies. Nat. Rev. Drug Discov., 12, 147-168 (2013).

Durie, F.H., T.M. Foy, S.R. Masters, J.D. Laman and R.J. Noelle: The role of CD40 in the regulation of humoral and cell-mediated immunity. Immunol. Today, 15, 406-11 (1994).

Flaherty, D.K.: Immunology for pharmacy. Elsevier/Mosby 2012. http://doi.org/10.1016/C2009-0-40304-0 (2012).

Fournel, S., S. Wieckowski, W. Sun, N. Trouche, H. Dumortier, A. Bianco, O. Chaloin, M. Habib, J.C. Peter, P. Schneider, B. Vray, R.E. Toes, R. Offringa, C.J.M. Melief, J. Hoebeke and G. Guichard: C3symmetric peptide scaffolds are functional mimetics of trimeric CD40L. Nat. Chem. Biol., 1, 377-382 (2005).

Franz, A.K.: The synthesis of biologically active organosilicon small molecules. Curr. Opin. Drug. Disc., 10, 654-671 (2007).

Galloway, W.R., A. Isidro-Llobet and D.R. Spring: Diversity-oriented synthesis as a tool for the discovery of novel biologically active small molecules. Nat. Commun., 1, 80 (2010).

Goodsell, D.S. and A.J. Olson: Structural symmetry and protein function. Annu. Rev. Cell. Dev. Biol., 29,105-153 (2000).

Guarnieri, F.: Designing a small molecule erythropoietin mimetic. In: Fragment-Based Methods in Drug Discovery, Methods in Molecular Biology (Ed.: A. Klon). Humana Press, NY, 1289, 185$210(2015)$

Herodin, F., P. Thullier, D. Garin and M. Drouet: Nonhuman primates are relevant models for research in hematology, immunology and virology. Eur. Cytokine Netw., 16, 104-116 (2005).

Imai, A., T. Suzuki, A. Sugitani, T. Itoh, S. Ueki, T. Aoyagi, K. Yamashita, M. Taniguchi, N. Takahashi, T. Miura and T. Shimamura: A novel fully human anti-CD40 monoclonal antibody, 4D11, for kidney transplantation in cynomolgus monkeys. Transplant, 84, 1020 1028 (2007).

Karpusas, M., Y. M. Hsu, J. H. Wang, J. Thompson, S. Lederman, L. Chess and D. Thomas: $2 \AA$ crystal structure of an extracellular fragment of human CD40 ligand. Structure, 3, 1031-1039 (1995).
Kawabe, T., M. Matsushima, N. Hashimoto, K. Imaizumi and Y. Hasegawa: CD40/CD40 ligand interactions in immune responses and pulmonary immunity. Nagoya. J.Med. Sci., 73, 69-78 (2011).

Kitagawa, M., D. Goto, M. Mamura, I. Matsumoto, S. Ito, A. Tsutsumi and T. Sumida: Identification of three novel peptides that inhibit CD40-CD154 interaction. Mod. Rheumatol., 15, 423-426 (2005).

Kooten, C.V. and J. Banchereau: CD40-CD40 ligand: A multi-functional receptor-ligand pair. Adv. Immunol., 61, 1-78 (1996).

Koyama, I., T. Kawai, D. Andrews, S. Boskovic, O. Nadazdin, S.L. Wee, H. Sogawa, D.L. Wu, R.N. Smith, R.B. Colvin and D.H. Sachs: Thrombophilia associated with anti-CD154 monoclonal antibody treatment and its prophylaxis in nonhuman primates1. Transplant, $77,460-462$ (2004).

Krieger, E., S.B. Nabuurs and G. Vriend: Homology modeling. Methods Biochem. Anal., 44, 509-524 (2003).

Liao, S.M., Q.S. Du, J.Z. Meng, Z.W. Pang and R.B. Huang: The multiple roles of histidine in protein interactions. Chem. Cent., J., 7, 44 (2013).

Morris, J.S., C.D. Frith, D.I. Perrett, D. Rowland, A.W. Young, A.J. Calder and R.J. Dolan: A differential neural response in the human amygdala to fearful and happy facial expressions. Nature, 383 , 812-815(1996).

Morris, J.S., K.J. Friston, C. Büchel, C.D. Frith, A.W. Young, A.J. Calder and R.J. Dolan: A neuromodulatory role for the human amygdala in processing emotional facial expressions. Brain, 121, 47-57 (1998).

Park, H., S. Lee, S. Lee and S. Hong: Structure-based de novo design and identification of D816V mutant-selective c-KIT inhibitors. Org. Biomol. Chem., 12, 4644-4655 (2014).

Patnaik, B.B., H.J. Hwang, S. Baliarsingh, J.M. Chung, M.K. Sang, H.R. Min, J.E. Park, H.C. Cho, S.W. Kang, S.Y. Park, H.S. Park, J.S. Lee and Y.S. Lee: In silico characterization of single and tandem repeat galectin from terrestrial slug, Incilaria fruhstorferi. J. Environ. Biol., 40, 940-947 (2019).

Roman, G.: Mannich bases in medicinal chemistry and drug design. Eur. J. Med. Chem., 89, 743-816 (2015).

Sievers, F., A. Wilm, D. Dineen, T.J. Gibson, K. Karplus, W. Li, R. Lopez, H. McWilliam, M. Remmert, J. Söding, J.D. Thompson and D.G. Higginsa: Fast, scalable generation of high-quality protein multiple sequence alignments using Clustal Omega. Mol. Sys. Biol., 7, 539 (2011).

Silvian, L.F., J.E. Friedman, K. Strauch, T.G. Cachero, E.S. Day, F. Qian, B. Cunningham, A. Fung, L. Sun, L. Su and Z. Zheng: Small molecule inhibition of the TNF family cytokine CD40 ligand through a subunit fracture mechanism. ACS Chem. Biol., 6, 636-647 (2011).

Singh, J., E. Garber, H.V. Vlijmen, M. Karpusas, Y.M. Hsu, Z. Zheng, D. Thomas and J.H. Naismith: The role of polar interactions in the molecular recognition of CD40L with its receptor CD40. Prot. Sci., 7, 1124-1135 (1998).

van Kooten, C. and J. Banchereau: CD40-CD40 ligand. J. Leukoc. Biol., $67,2-17(2000)$.

Vani, V. and M. Sivanandham: Computational design of small molecules that mimic CD40L. Int. J. Comput. Intell. Hlth. Care Inform., 1, 68$75(2008)$.

Vani, V., S. Prasad and M. Sivanandham: CD40 Ligand mimetic molecules activate B-cell proliferation and immunoglobulin isotype switching. Int. J. Pharm. Chem. Biol. Sci., 4, 640-647 (2014).

Venselaar, H., E. Krieger and G. Vriend: Homology modeling. In: Structural Bioinformatics (Eds.: J. Gu and P.E. Bourne) WileyBlackwell: Hoboken, NJ, pp.715-732 (2009).

$\mathrm{Yi}, \mathrm{Z}$. and G.A. Bishop: Regulatory role of CD40 in obesity-induced insulin resistance. Adipocyte, 4, 65-69 (2015). 